\title{
The Influence and Application of Aesthetic Art on Garden Landscape Design
}

\author{
Zhenlin Li \\ Chengdu Agricultural College, Chengdu Sichuan, 611130, China
}

Key words: landscape art, Garden, Aesthetics, Design.

\begin{abstract}
Aesthetic art does not depend on utilitarianism or truth, goodness, and it is a theoretical branch of philosophy. The sensation of beauty involves spiritual level, material level, rational level and perceptual level, which can keep people away from hustle and bustle, and return to quiet and harmonious life. Art design should be based on aesthetics, make the design program full of vitality, highly integrate the beauty of virtue and form, and enhance the infinite natural landscaping effect. Landscape may be influenced by aesthetic art. Landscape design aesthetics is concerned about the beauty of form, technique, material and function, including form feelings, meaning experience and value recognition, aimed to change material appearance and release aesthetic art spirit, integrate aesthetic value and functions, creativity and perceptibility. This paper explores the influence of the present aesthetic art on the landscape design and the practice of aesthetic art, to clarify the aesthetic standard and aesthetic law of landscape design.
\end{abstract}

\section{Introduction}

Garden is a four-dimensional space works of art, showing aesthetic spirit and cultural spirit. The space-time art can be continuously changed and re-broadcast, and the artistic conceptional space is dynamically changing, which may provide spiritual enjoyment, with leisure, aesthetics, workability and practicality. Aesthetic spirit is closely related with artistic trends, political changes and economic changes. The art of garden aesthetics originated from the combination of nature and spirit, which can generate intelligence and happiness, promote the desire to be adjusted, and then feel comfortable and healthy. Therefore, it is a kind of dynamic and open aesthetic value. The landscape is influenced by aesthetic art and fully presents aesthetic spirit ${ }^{[1]}$. In the design of the landscape, we need to go beyond the physiological need and visual perception, integration of the ecology, geography, philosophy and other aesthetic paradigm, innovative design based on cultural accumulation, life experience, experience memory, basic perception, proper treatment of the relationship between design elements and the landscape, to create the landscape meeting the public aesthetic taste.

\section{Influence}

\section{Influence of ecological aesthetics art}

Ecological aesthetics emphasizes harmony between man and nature, and the ecological system as an organic whole. Ecological aesthetics is closer to the real life, breaks through the framework of artistic philosophy, and aesthetic ideas have penetrated into urban construction, environmental protection, ecological protection and other fields, and had an influence on the landscape design. Under the influence of ecological aesthetics, the landscape design emphasizes the concept of symbiosis ecological philosophy, follows the principle of generality and organicity, pursues the ecological beauty and environmental beauty, and creates ecology, low-carbon and green landscape design. Landscape design is subject to the impact of ecological aesthetics, mainly because of quick pace of 
modern social life, deterioration of the environment, changes of living space, and people's desire to return to the humanity and nature [2]. Ecological aesthetic art attaches great importance to dealing with human-land relationship, and the landscape design can establish the human-land relationship, and get a sense of belonging in the ecological culture. For example, in Figure 1, the "happy countryside" landscape is composed of winding wooden footways and the gourd vine fully crawls on the three-dimensional pergola made of bamboo. The happy pastoral landscape life cycle is consistent with the laws of nature, so that people can feel the beauty of four seasons in the blossom or fruit of plants. The theoretical basis of ecological aesthetics is mainly natural value and ecological ethics. Under the influence of ecological aesthetics, the landscape design needs to integrate the ecological function and aesthetic function. Fully consider the surrounding environment, water source, soil, topography, terrain and environmental capacity and other factors in the design of lighting systems, water works, lake and hills, bridges, roads and accessorial buildings, to protect the ecological system, and allow people to feel and care for nature when they enjoy the landscape [3].

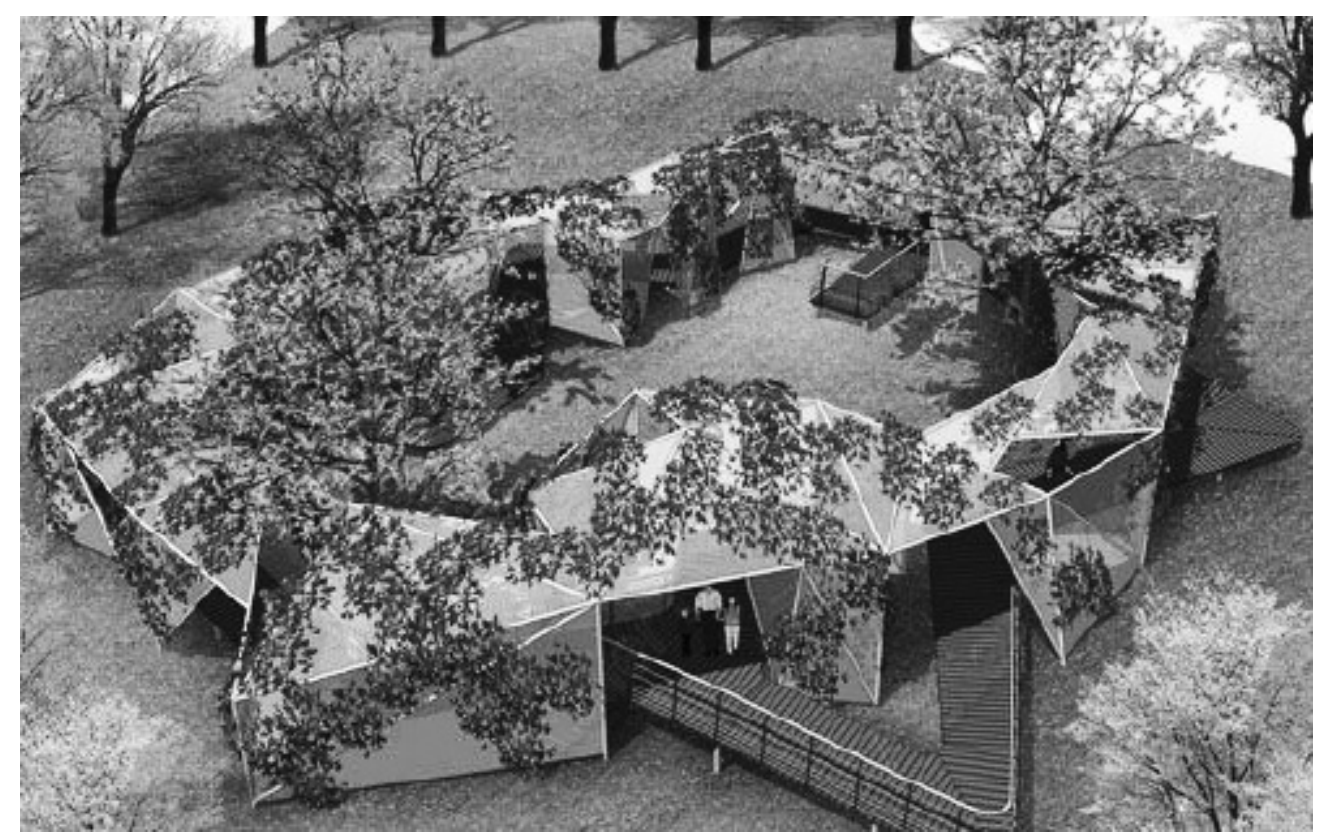

Fig. 1

\section{Influence of classical aesthetic art}

The aesthetic art of classical gardens is closely related to the traditional culture. Under the influence of classical aesthetics art, the landscape design advocates the natural landscape, and creates picturesque scene and artistic conception. The nature-admiring aesthetic idea contains the unity of heaven and man. Landscape design is not only the process of creating garden, but also the process of spiritual creation. The design of the landscape integrates culture, calligraphy and painting, uses rockery and water to imitate the nature, and uses stone inscription, plaque or couplet to reflect landscape culture, to pursue the integration of affections and landscape, and create "one stone, full of mood; one mountain, full of scenes", to enable visitors to associate with the landscape, and also increase the cultural connotation and significance. The landscape space curve is flowing, or consistent, to create the scene of the path winding through high peaks ${ }^{[4]}$. For example, the pavilion in Figure 2, "Flowing Water", is associated with ancient artisans reciting poems and drinking together, which can stimulate imagination and express spiritual needs. In order to create a classical aesthetic mood, the design of garden landscape usually needs the help of winding path, rare rocks, etc., and common accessorial buildings include various types of pavilions, cooperating with flowing water, stone, rockery and others. The corridor may go through the house. The landscape, full of rhythmic beauty, emphasizes curves, and scenes changes, taking lattice window or rockery as a line of sight 
barrier. The landscape panorama can be shown after several twists and turns by clever use of separation of trees, landscapes or building, so that landscape changes consistently, and change cooperates with convergence ${ }^{[5]}$. For example, in Figure 3, the landscape design makes full use of rocks, trees and pavilions to create an independent space environment and the connection present unique poetic images in the rocks and trees.

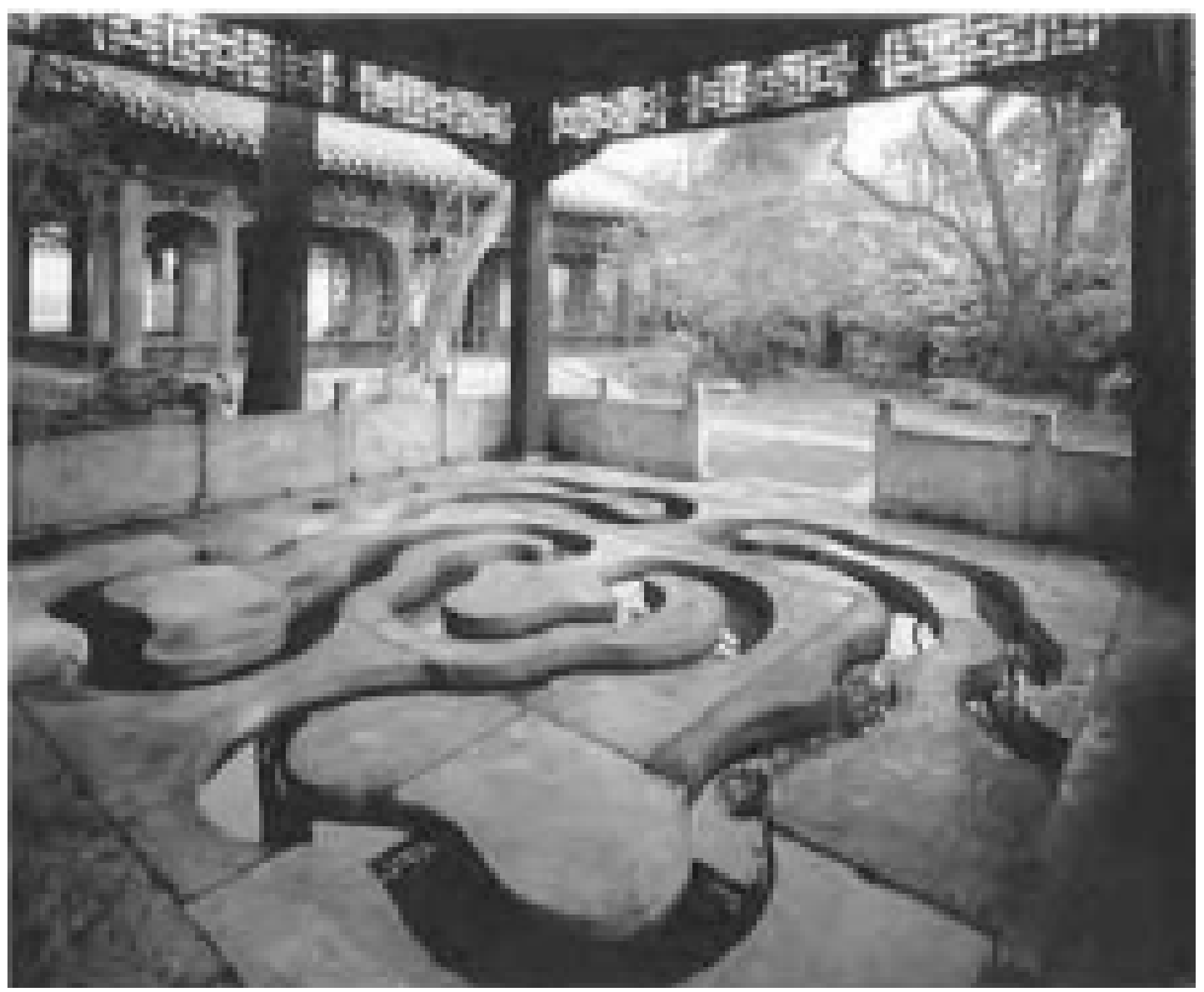

Fig. 2 


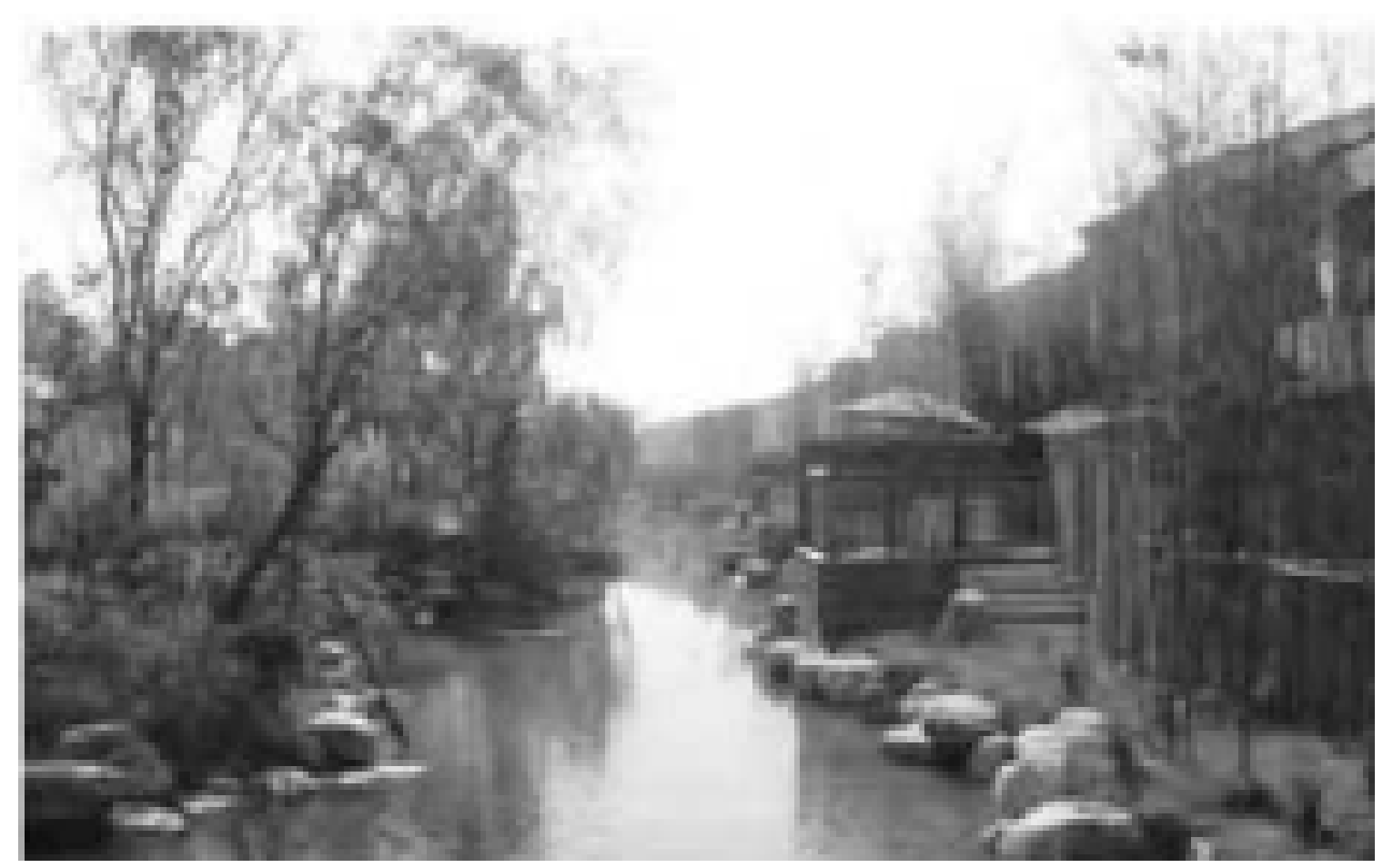

Fig. 3

\section{Influence of technical aesthetic art}

Technical art belongs to applied aesthetics, properly combining material needs and spiritual needs, and advocating the combination of aesthetic theory and technical science. Technical aesthetics has close contact with industrial aesthetics, mainly studying the beauty of structure, material and geometric figure, first used in the field of commodity exchange or industrial product design. Material design adopts advanced science and technology, and also requires the use of abstract art form, artistic law and aesthetic consciousness to create beauty. At present, technical aesthetics has penetrated into the field of landscape design, and has had a profound impact on landscape design and broadened time dimension and spatial dimension of landscape design ${ }^{[6]}$. Under the influence of technical aesthetics, design are weakening the harmonious beauty and sensibility beauty of landscape, but emphasizing the landscape abstract image or the independence of the landscape in the environmental space, the intersection of landscape color and material texture, to create more active environmental space for the landscape. Technical aesthetics can also make the landscape design thinking divergent, which means that the landscape is no longer as an independent aesthetic image, but able to produce a border effect or the relative isolation with other landscapes or the environment, so as to enhance the aesthetic significance of space and spiritual vitality, cultural charm of the landscape based on the design concept of interwoven fusion and openness. For example, the landscape in Figure 4 combines the tensioning membrane technology, and uses fiber canvas and stakes to design a surreal sculpture landscape. In the ups and downs of tents, cactus and linen emerge, and the garden is surrounded by wood. Three-dimension space designed by technical aesthetic is layering, to the beauty of structural form, strength and simplification. 


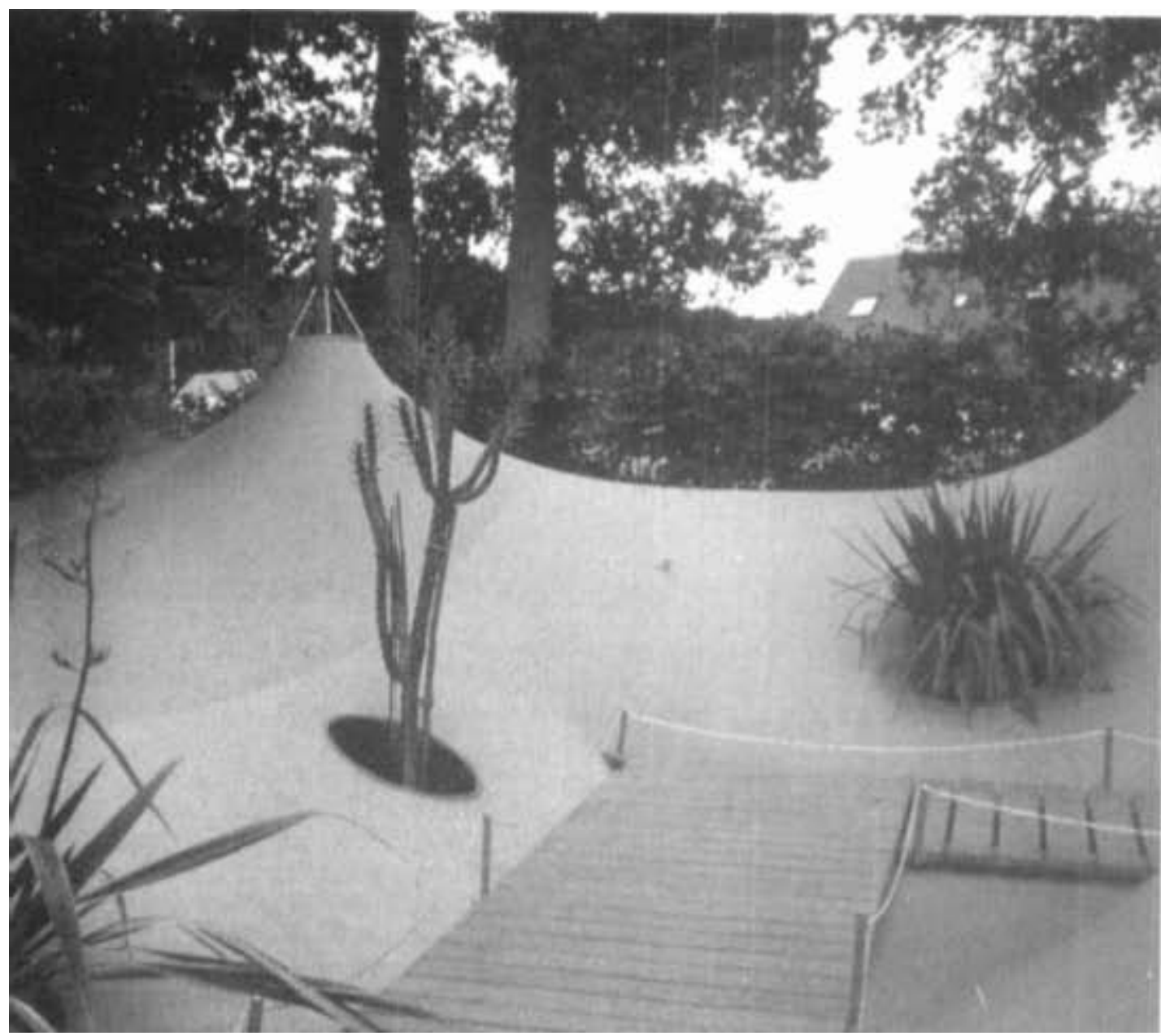

Fig. 4

\section{Influence of accepting aesthetics art}

Accepting aesthetics was originally used to analyze literary works, mainly studying whether the reader accepted the work and the reaction to the work. At present, accepting aesthetics has broken the shackles of traditional literary applications and widely influenced other art disciplines. Accepting aesthetic art emphasizes that the end of the creative process does not mean that the final completion of works of art and realization of the value of artistic works depends mainly on the audience acceptance. Landscape design is influenced by the art of accepting aesthetics. Under the guidance of accepting aesthetic art concept, the landscape design scheme can be regarded as literary works, and the audience of the landscape as the reader. The degree of acceptance and recognition is the decisive factor on whether the landscape design is reasonable. In the landscape design stage, it is necessary to feel life, watch life, embrace life and think about life, to enrich aesthetic experience and aesthetic feeling, to integrate personal experience, personal expertise, artistic taste, and life experience into the design, so as to achieve the landscape design unifying ration and emotion, nature and phenomenon, object and subject, and creating aesthetic images in line with the aesthetic needs of the public ${ }^{[7-8]}$. The design may keep appropriate blank, so that audiences can fly their imagination, and feel the aesthetic characteristics with their heart. For example, in Figure 5, in the garden square green belt, shade chairs are provided, for the eyes of viewers, but also for the rest of viewers, easy to be recognized and accepted, and in line with the aesthetic design principles. 


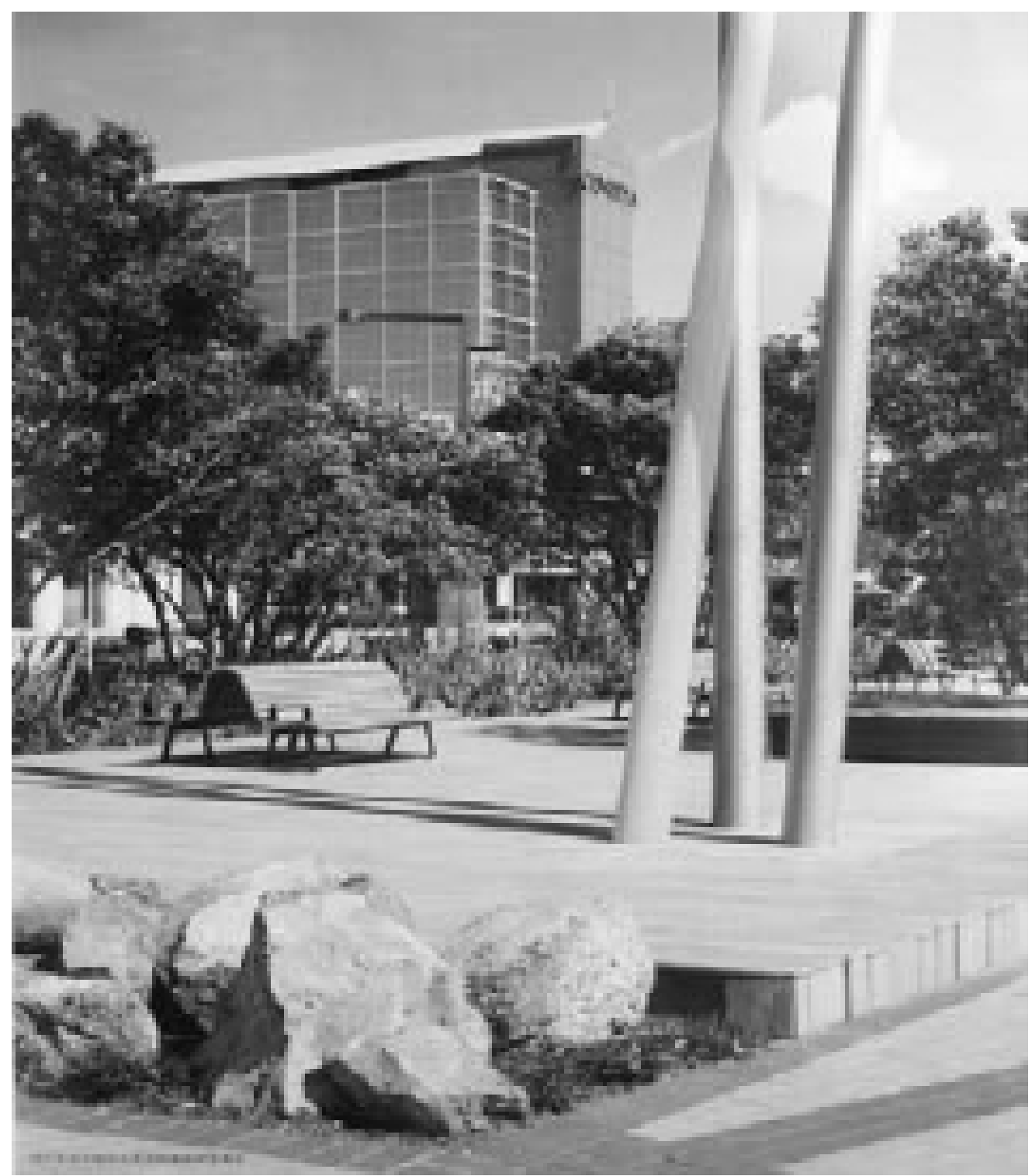

Fig. 5

\section{Application}

\section{Design background}

A garden landscape project is the leisure center and cultural center of the district, with investment and construction funds of about 500 million Yuan. The garden sets up artificial island, and has four waters, green area of about $20 \mathrm{hm}^{2}$, road area of about $2.5 \mathrm{hm}^{2}$, and water area of about $90 \mathrm{hm}^{2}$. The traffic conditions are preferable, able to echo with other scenic areas. In order to ensure that the garden can give full play to recreational function, the aesthetic art is adopted to improve the landscape design.

\section{Design scheme}

\section{Design of waters}

In the garden, around "ancient waterside" landscape, meandering streams are design, and pavement material adopts simple, generous, and practical quartzite. Interior pavement design uses the form of ice crack, so that the courtyard twists and turns deep, simple and elegant, ethereal and transparent, away from the hustle and bustle, to create the ancient mood. While next to the water, plant bamboos and reeds, and set the rockery, to show the natural beauty. In the terrain with large height difference, 
design water terraces. Stacked waves of water terraces can be reflected on lush trees on the mountain, so that the water scene features vividness. Along the bank of the lake, design hydrophilic facilities, including the pier, hydrophilic square, boat house, water pavilion, lake promenade and hydrophilic platform. Hydrophilic facilities are practical, and in these places, visitors can have a rest, breath moist and fresh air and enjoy the water scenes. Hydrophilic facilities make revetment lines become richer, able to beautify the water landscape environment. In the water scenes, set various types of stones. Stepping stones are diamond-shaped, and mural stones are block. On the stone murals, engrave famous words and sentences, to add spirit to the landscape, so that visitors can perceive the inward nature of mountains and waters, and cultivate their sentiment. Mural paintings include double-phoenix, double-dragon, and dragon-phoenix, implying the prosperity, to integrate humanistic connotation into the natural ecological landscape, such as grass and water.

Design of green plants

On both sides of the road in the garden, plant shade trees. In the open space, plant herbs, shrubs and trees. Cooperate with fruit plants, flowering plants, and foliage plants, combine with deciduous shrubs, trees and evergreen shrubs, trees, herbs such as siberian wildrye, and leymus chinensis. When planting, pay attention to the space of planting, and stick to the principle of natural law to create a natural beauty. Use various design methods, such as corridor hanging flowers, winding paths, view borrowing and blocking, and flower wall and leakage window, to well integrate with the other landscape and plant. Orchids, and narcissus help create elegant mood, and chrysanthemums has a hidden mood. Plants with some wonderful smell can make four seasons fragrant. Locust trees, willows, and lotus, etc., can create sounds of nature, such as cicadas singing in high locust tree, orioles singing in the willows, rain drops on withered lotus, whistling of the wind in the pines and bamboos, etc., to reduce the noise in the garden, make the garden environment quiet and elegant. For winter landscape, keep green pine and cypress, and plant evergreen plants, such as pinus sylvestris; for autumn landscape, plant cattail, northeast birch, etc.; for summer landscape, plant aspen, lespedeza, caryophyllus, etc. to create green and heavy shade; for spring landscape, plant cuckoo, pearl plum, bougainvillea and syzygium aromaticum and so on to make a flowery and colorful spring. In order to maintain the ecological balance, in wetlands, plant water lilies, lotus, sedge, nymphoides, zanthoxylum, water onion and cattail, and plant azalea, oleander, wood hibiscus and weeping willow in shallow water and at the bank of wetland. The combination of virtuality and reality enriches landscape and maintains the diversity of biological species. On some lands in the garden, mix broadleaf plants and coniferous plants, to imitate virgin forest landscape, improve the greening rate, and optimize air quality. For the solemn space in the garden, plant simple and vigorous, tall and straight fir, cypress, pine and other plants.

\section{Conclusions}

In conclusion, the landscape aesthetic highly integrates the beauty of society, art and nature. Aesthetics art can produce purely objective, purely subjective or subjective and objective influence on landscape design, which reflects the subjective feelings of aesthetic subjects. Landscape space creation, spatial organization form embodies the profound aesthetic way of thinking. As space consciousness, cosmic consciousness, philosophical concept is different, the influence also changes. Natural beauty, artificial beauty, artistic beauty, and form beauty have their own characteristics. In the practice of aesthetic art in landscape design, we should develop the inherent nature of human beings, pursue and explore aesthetic art, combine rules and natural aesthetics concept, integrate with aesthetic art, use nature or transform nature, and create ideal leisure surroundings in the garden. When designing the landscape, we should consider the background of different aesthetic art, including the class, culture, region and times, improve the aesthetic consciousness, aesthetic ability and aesthetic experience, extract the core orientation of aesthetic value, and design different styles of landscape under the guidance of aesthetic value. 


\section{References}

[1] Gu Kai. Historical Development and Form Concern of the "Picturesque" Appreciation and Construction in Chinese Landscape-Comment on "Two Picturesque Aesthetic Concepts and Gardens, Journal of Architecture, 2016 (9): 57-61.

[2] Yang Xin, Zhang Qi. Form Language Change under Rational and Overall Design ThinkingHeritage and Development of French Modern Garden on Classical Garden, Huazhong Architecture, 2016 (3): 22-26.

[3] Chen Zhengchuan, Yuan Jiafan. Application of Sponge City Concept in Landscape Design - A Case Study of South Sub-center of State Grid Service Center, Architecture and Culture, 2017 (1): 14-23.

[4] Liu Hailong, Ning Qiuling, Zhao Dongbo. Theoretical Analysis and Practice of Beijing Royal Garden Construction - Taking the Construction of Beijing Park of the Third Green Expo as an Example, Land Greening, 2017 (1): 51-53.

[5] Gao Fei, Zhang Jing. North-South Cultural Integration and Poetic Image Construction-Folk Culture of Meaning of Jining Ancient Garden, Folklore Study, 2016 (1): 129-135.

[6] Li Xiaoqi. The Influence of Tang Dynasty Poems on the Construction of Garden Space Art in Song Dynasty - Prose About Garden of Song Dynasty, Jinan Journal (Philosophy and Social Sciences), 2016 (4): 64-71.

[7] Xiao Jing. Landscape Visual Reappearance of Architecture based on the Text in Ming Dynasty -Taking the "Interleaved Ancient Woods" in Lingering Garden as an Example, Journal of Architecture, 2016 (1): 31-35.

[8] Cai Qiuyang, Gao Chi. Analysis on Influencing Factors and Mechanism of Tourists' Satisfaction in Garden Expo Park-based on Empirical Study of Structural Equation Model, China Garden, 2016 (8): 58-64. 